\title{
Classification des Représentations Tempérées d'un Groupe p-Adique non Connexe
}

\author{
Karem Bettaïeb
}

Received: 6 May 2009 / Accepted: 5 June 2011 / Published online: 27 September 2011

(C) The Author(s) 2011. This article is published with open access at Springerlink.com

Résumé Soit $G$ le groupe des points définis sur un corps p-adique d'un groupe réductif non connexe. Dans cette note on prouve que toute représentation irréductible tempérée de $G$ est irréductiblement induite d'une essentielle d'un sous-groupe de Lévi cuspidal de $G$.

\begin{abstract}
Let $G$ be the group of points defined over a $p$-adic field of a nonconnected reductive group. In this note, we prove that every tempered irreducible representation of $G$ is irreducibly induced from an essential one of a cuspidal Levi subgroup of $G$.
\end{abstract}

Keywords Groupes p-adiques • Représentations • Sous-groupes de Levi

Mathematics Subject Classifications (2010) $11 \mathrm{E} 95 \cdot 20 \mathrm{G} 05 \cdot 20 \mathrm{G} 15$

\section{Introduction}

Soit $G$ l'ensemble des points rationnels d'un groupe algébrique réductif non connexe p-adique de caractéristique 0 . Notons $\mathcal{V}(G)$ l'ensemble des caractères virtuels tempérés de $G$, c'est-à-dire que $\mathcal{V}(G)$ est l'ensemble des combinaisons linéaires finies des caractères des représentations irréductibles tempérées de $G$. On dit que l'élément

Presented by Alain Verschoren.

K. Bettaïeb $(\varangle)$

Laboratoire de Théorie des Groupes, Représentations-Applications,

Institut de Mathématiques de Jussieu, 175, Rue de Chevaleret, 75013 Paris, France

e-mail: bettaieb@math.jussieu.fr 
$\Theta \in \mathcal{V}(G)$ est un caractère virtuel supertempéré $[9,11]$ s'il satisfait à la condition suivante:

Pour tout sous-groupe de Cartan $T$ de $G$ et pour toute constante positive $n$, on a:

$$
\sup _{t \in T^{\prime}}\left|D_{G}(t)\right|^{1 / 2}|\Theta(t)|\left(1+\sigma_{*}(t)\right)^{n}<\infty
$$

où $D_{G}$ est le facteur discriminant usuel, $\sigma_{*}$ estime la croissance sur $G / A_{G}, T^{\prime}=$ $T \cap G^{\prime}$ où $G^{\prime}$ est l'ensemble des éléments $x \in G$ tels que $D_{G}(x) \neq 0$.

Notons $\mathcal{V}_{s t}(G)$, le sous-espace vectoriel des éléments supertempérés de $\mathcal{V}(G)$. On sait que le caractère d'une représentation irréductible tempérée $\pi$ est supertempéré si et seulement si $\pi$ appartient à la série discrète [11].

Comme résultat préliminaire, et à l'aide des travaux de J. Arthur [1] et R. Herb [11], on démontre que tout élément de $\mathcal{V}(G)$ s'écrit comme combinaison linéaire finie d'induites de caractères virtuels supertempérés (Théorème 4). Soient $\Theta_{i} \in$ $\mathcal{V}_{s t}\left(L_{i}\right)$, où $L_{i}(i=1,2)$ sont deux sous-groupes de Lévi de $G$. On note $i_{G, L_{i}}\left(\Theta_{i}\right)$ le caractère induit de $\Theta_{i}$. Par analogie avec les caractères cuspidaux, on démontre que $i_{G, L_{1}}\left(\Theta_{1}\right)=i_{G, L_{2}}\left(\Theta_{2}\right)$ si et seulement si, il existe $t \in G$ tel que $L_{1}=t L_{2} t^{-1}$ et $\Theta_{1}=t \Theta_{2}$ (Proposition 6) . Ce résultat nous permettra de déduire (Corollaire 7) qu' à chaque élément $\Theta \in \mathcal{V}(G)$ correspond, modulo la conjugaison par $G$, une famille finie unique $\left\{\left(L_{i}, \Theta_{i}\right)\right\}_{1 \leq i \leq p}$, où $\Theta_{i} \in \mathcal{V}_{s t}\left(L_{i}\right)$ et $L_{i}$ sous-groupes de Lévi de $G$ tel que:

$$
\Theta=\sum_{1 \leq i \leq p} i_{G, L_{i}}\left(\Theta_{i}\right)
$$

Notons $P_{t}=M_{t} N_{t}$ le sous-groupe parabolique de $G$ correspondant à l'élément semi-simple $t$ de $G$ [6] et $G_{c}$, "la partie compacte" de $G$, constituée des éléments semi-simples $t$ de $G$ tel que $P_{t}=G$.

Comme propriété des caractères virtuels supertempérés, on montre que pour tout $\Theta \in \mathcal{V}_{s t}(G)$ et $f$ une fonction dans l'espace de $\operatorname{Schwartz} \mathcal{C}(G)$, de $G$ :

$$
<\Theta, f>:=\int_{G} \Theta(x) f(x) d x=\int_{G_{c}} \Theta(x) f(x) d x
$$

“La trace compacte” de $\Theta \in \mathcal{V}(G)$ est définie par:

$$
<\Theta, f>_{G, c}:=\int_{G_{c}} \Theta(x) f(x) d x \quad f \in \mathcal{C}(G)
$$

L. Clozel [4] a montré que la trace d'une représentation admissible de longueur finie se décompose en somme de trace compacte des modules de Jacquet normalisée. Par analogie, on démontre, qu'étant donné $\Theta \in \mathcal{V}(G)$ et $f \in \mathcal{C}(G)$, il existe une famille finie $\left\{\left(L_{i}, \Theta_{i}\right)\right\}_{1 \leq i \leq p}$ de caractères virtuels supertempérés de $G$, unique modulo la conjugaison par $G$, telle que:

$$
<\Theta, f>=\sum_{1 \leq i \leq p}<\Theta_{i}, f_{Q_{i}}>_{L_{i}, c}
$$

où $f_{Q_{i}} \in \mathcal{C}\left(L_{i}\right)$ est le terme constant de $f$ le long d'un sous-groupe parabolique $Q_{i}$ ayant pour sous-groupe de Lévi $L_{i}$ (Corollaire 11). Cette égalitée montre, en particulier, que la trace d'une représentation tempérée irréductible se décompose en somme finie de "trace compacte". 
Notons $\Pi(G)$, l'ensemble des classes d'équivalence des représentations irréductibles tempérées de $G$. Une représentation dans $\Pi(G)$ est dite elliptique si son caractère est non nul sur l'ensemble régulier elliptique de $G$. Notons par $\Pi_{\text {ell }}(G)$ l'ensemble des classes d'équivalence des représentations elliptiques de $G$. De même, une représentation irréductible tempérée de $G$ est dite essentielle (ou limite de série discrète [5]) si elle n'est pas proprement irréductiblement induite par induction parabolique. Notons $\Pi_{e s s}(G)$ l'ensemble (des classes d'équivalence) des représentations essentielles de $G$. On sait que $\Pi_{\text {ell }}(G) \subset \Pi_{\text {ess }}(G)$ et que si $G=S O(n)$ alors $\Pi_{\text {ess }}(G)$ contient strictement $\Pi_{\text {ell }}(G)[10]$.

On se donne $(M, \sigma)$ une paire discrète de $G$, c'est-à-dire que $M$ est un sousgroupe de Lévi de $G$ et $\sigma$ une représentation irréductible de $M$, de carré intégrable modulo la composante déployée $A_{M}$ du centre de $M$. Notons $i_{G, M}(\sigma)$ la classe de la représentation induite $\mathcal{I n d}_{P=M N}(\sigma)$ et $\Re_{\sigma}:=\Re_{\sigma}^{G}$ le $\Re$-groupe correspondant. C'est un groupe fini ayant la propriété que l'algèbre commutante de $i_{G, M}(\sigma)$ est isomorphe à $\mathbb{C}\left[\Re_{\sigma}\right]_{\eta_{\sigma}}$ l'algèbre du groupe $\mathfrak{R}_{\sigma}$ tordue par un cocycle $\eta_{\sigma}$ (voir [1], §2). Notons $\mathfrak{a}_{M}$ (resp. $\mathfrak{a}_{G}$ ), l'algèbre de Lie réelle de la composante déployée de $M$ (resp. $G$ ). Pour tout $r \in \Re_{\sigma}$, posons:

$$
\mathfrak{a}_{M}^{r}:=\left\{H \in \mathfrak{a}_{M}, \quad r H=H\right\} \quad \text { et } \quad \mathfrak{a}_{M}^{\Re_{\sigma}}:=\bigcap_{r \in \Re_{\sigma}} \mathfrak{a}_{M}^{r}
$$

On dit que le groupe $\mathfrak{R}_{\sigma}$ est essentiel si $\mathfrak{a}_{M}^{\mathfrak{R}_{\sigma}}=\mathfrak{a}_{G}$.

Comme conséquence du Corollaire 7, on démontre qu'une composante irréductible de $i_{G, M}(\sigma)$ est essentielle si et seulement si le groupe $\Re_{\sigma}$ est essentiel. Ainsi, on aura démontré la conjecture de Clozel [5]: si $\pi$ est essentielle alors toutes les reliées à $\pi$ le sont aussi (une représentation irréductible tempérée $\pi_{2}$ de $G$ est dite reliée à $\pi_{1}$ si $\pi_{1}$ et $\pi_{2}$ proviennent d'une même paire discrète $(M, \sigma)$ de $G$ [12]).

Cette classification des représentations irréductibles essentielles de $G$, nous permet de montrer une autre conjecture de Clozel [5]: toute représentation irréductible $\pi$ de $G$ est irréductiblement induite d'une essentielle. De plus, si $\pi=i_{G, L_{1}}\left(\delta_{1}\right)=$ $i_{G, L_{2}}\left(\delta_{2}\right), \delta_{i} \in \Pi_{\text {ess }}\left(L_{i}\right)$ et $L_{i}$ sous-groupes de Lévi de $G$, alors il existe $t \in G$ tel que $L_{1}=t L_{2} t^{-1}$ et $\delta_{1}=t \delta_{2}$.

\section{L'Espace des Caractères Virtuels $\mathcal{V}(G)$}

Dans ce paragraphe on montre que tout caractère virtuel tempéré s'écrit comme combinaison linéaire d'induites de caractères virtuels supertempérés.

Soit $\mathbf{G}$ un groupe algébrique connexe réductif défini sur un corps local non archimédien $F$ de caractéristique 0 . Notons $G(F)$ l'ensemble des points $F$-rationnels de G. Dans la suite on notera $G:=G(F)$.

Pour la suite, on fixe une composante de Lévi $F$-rationnelle $M_{0}$ d'un certain sousgroupe parabolique minimal $P_{0}$ de $\mathbf{G}$ défini sur $F$. On pose $W_{0}^{G}:=N_{G}\left(M_{0}\right) / M_{0}$ où $N_{G}\left(M_{0}\right)$ est le normalisateur de $M_{0}$ dans $G$.

Par définition, un groupe de Lévi $M$ de $G$ est un sous-groupe algébrique de $G$ contenant la composante de Lévi $M_{0}$ d'un certain sous-groupe parabolique défini sur $F$.

Si $M$ est un sous-groupe de Lévi de $G$ de composante déployée $A_{M}$, soit $\mathcal{L}(M)$ l'ensemble des sous-groupes de Lévi de $G$ contenant $M$ et $\mathcal{L}_{0}(M)=$ 
$\{L \in \mathcal{L}(M) \mid L \neq G\}$. Si $L \in \mathcal{L}$, on note $\operatorname{par} \mathcal{L}^{L}(M)$ l'ensemble des sous-groupes de Lévi de $L$ contenant $M$.

Si $M=M_{0}$, on écrit $\mathcal{L}:=\mathcal{L}\left(M_{0}\right), \mathcal{L}_{0}:=\mathcal{L}_{0}\left(M_{0}\right)$ et $A_{0}:=A_{M_{0}}$.

Soit $\pi$ représentation admissible de $G$, notons par $\mathcal{A}(\pi)$ l'ensemble de toutes les combinaisons linéaires finies des coefficients matriciels de $\pi$ et $\mathcal{A}(G)=\bigcup_{\pi} \mathcal{A}(\pi)$, la réunion étant prise sur toutes les représentations admissibles de $G$. Pour tout sous-groupe parabolique $P=M N$ de $G$ et $f \in \mathcal{A}(G)$, il existe un terme constant $f_{P} \in \mathcal{A}(M)$ ayant la propriété $[13,2.6]$ : soit $m \in M$, il existe une constante positive $c$ telle que:

$$
\delta_{P}(m a)^{1 / 2} f(m a)=f_{P}(m a) \quad \text { pour tout } a \in A_{M}^{+}(c)
$$

où $\delta_{P}$ est la fonction modulaire de $P$ et

$$
A_{M}^{+}(c)=\left\{a \in A_{M}: \quad|\alpha(a)| \geq c \quad \text { pout toute racine simple } \alpha \text { de }\left(P, A_{M}\right)\right\}
$$

La fonction $f_{P}$ est appelée terme constant de $f$ le long de $P$.

Soient maintenant $(\pi, V)$ une représentation admissible de $G$ et $P=M N$ un sousgroupe parabolique de $G$. Supposons que $\Theta:=\Theta_{\pi}$ est le caractère de $\pi$. Par analogie, on définit le terme constant $\Theta_{P}$ de $\Theta$ le long de $P$. Notons par $\bar{P}=M \bar{N}$ le sousgroupe parabolique opposé de $P, V(\bar{N}):=<\pi(\bar{n}) v-v, \bar{n} \in \bar{N}, v \in V>$ et $V_{\bar{N}}:=$ $V / V(\bar{N})$.

Soit $p: V \rightarrow V_{\bar{N}}$ la projection canonique. Pour tous $m \in M$ et $v \in V$, on définit:

$$
\pi_{\bar{N}}(m) p(v)=\delta_{\bar{P}}(m)^{-1 / 2} p(\pi(m) v)
$$

La représentation $\left(\pi_{\bar{N}}, V_{\bar{N}}\right)$ est appelée module de Jacquet normalisé de $(\pi, V)$ qui correspond à $\bar{P}$; on sait que c'est une représentation admissible de longueur finie de $M[13,2.3 .6]$. On pose $\Theta_{P}:=r_{\bar{P}, G}\left(\Theta_{\pi}\right)=\Theta_{\pi_{\bar{N}}}$ le caractère de $\pi_{\bar{N}}$. On appelle $\Theta_{P}$ le terme constant de $\Theta=\Theta_{\pi}$ le long de $P$.

Soient $f \in \mathcal{A}(G)$ et $P=M N$ un sous-groupe parabolique de $G$. Suivant [13, 3.1], on a: $f_{P}=\sum_{\chi} f_{P, \chi}$ où $\chi$ est un quasi-caractère de $A_{M}$. On pose:

$$
X_{f}\left(P, A_{M}\right):=\left\{\chi: f_{P, \chi} \neq 0\right\} \quad \text { et } \quad X_{\pi}\left(P, A_{M}\right)=\bigcup_{f \in \mathcal{A}(\pi)} X_{f}\left(P, A_{M}\right) .
$$

D’après [13, 3.3.1], on a:

$$
V_{\bar{N}}=\sum_{\chi \in X_{\pi}\left(P, A_{M}\right)} V_{\bar{N}, \chi} .
$$

Par conséquent:

$$
\Theta_{P}=\Theta_{\pi_{\bar{N}}}=\sum_{\chi \in X_{\pi}\left(P, A_{M}\right)} \Theta_{P, \chi}
$$

où $\Theta_{P, \chi}$ est le caractère de la restriction de $\pi_{\bar{N}}$ sur $V_{\bar{N}, \chi}$.

Notons $\Pi(G)$, l'ensemble de classes d'équivalence des représentations irréductibles tempérées de $G$. Si $\pi \in \Pi(G)$ et $P=M N$ un sous-groupe parabolique de $G$, on pose $X_{\pi}^{w}\left(P, A_{M}\right):=X_{\pi}\left(P, A_{M}\right) \cap \hat{A}_{M}$. D'après [13, 5.4.1.3], le quotient maximal tempéré de $\pi_{\bar{N}}$ est:

$$
\left(V_{\bar{N}}\right)^{w}=\sum_{\chi \in X_{\pi}^{w}\left(P, A_{M}\right)} V_{\bar{N}, \chi} .
$$


Soit $\Theta=\Theta_{\pi}$ le caractère de $\pi$, posons $\Theta_{P}^{w}:=\Theta_{\left(V_{\bar{N}}\right)^{w}}$ le caractère de $\left(V_{\bar{N}}\right)^{w}$. On appelle $\Theta_{P}^{w}$ le terme constant faible de $\Theta=\Theta_{\pi}$ le long de $P$.

Comme conclusion, on vient de définir pour tout sous-groupe parabolique $P=$ $M N$ de $G$ et $\pi \in \Pi(G)$, le terme constant $\left(\Theta_{\pi}\right)_{P}$ et le terme constant faible $\left(\Theta_{\pi}\right)_{P}^{w}$ de $\Theta_{\pi}$ le long du sous-groupe parabolique $P$.

On dit que $\Theta$ est un caractère virtuel de $G$ et nous écrivons $\Theta \in \mathcal{V}(G)$, s'il existe un nombre fini, $\pi_{1}, \pi_{2}, \ldots, \pi_{k} \in \Pi(G)$ et $c_{i} \in \mathbb{C}$ tels que:

$$
\Theta:=\sum_{1 \leq i \leq k} c_{i} \Theta_{\pi_{i}} .
$$

Ce qui nous permet de définir le terme constant $\Theta_{P}$ et le terme constant faible $\Theta_{P}^{w}$ de $\Theta$ le long d'un sous-groupe parabolique $P=M N$ de $G$ par:

$$
\Theta_{P}=\sum_{1 \leq i \leq k} c_{i}\left(\Theta_{\pi_{i}}\right)_{P} \quad \text { et } \quad \Theta_{P}^{w}=\sum_{1 \leq i \leq k} c_{i}\left(\Theta_{\pi_{i}}\right)_{P}^{w}
$$

Un élément $x \in G$ est dit régulier si $D_{G}(x) \neq 0$ où $D_{G}$ est le facteur discriminant standard défini dans $[13,4.7]$. Notons $G^{\prime}$ l'ensemble des éléments réguliers de $G$. Si $T$ est un sous-groupe de Cartan de $G$, on note $T^{\prime}:=T \cap G^{\prime}$.

Un élément $x \in G$ est dit elliptique si son centralisateur est compact modulo la composante déployée $A_{G}$ de $G$. Notons $G_{\text {ell }}$ l'ensemble des éléments réguliers elliptiques de $G$. Si $\Theta \in \mathcal{V}(G)$, on note $\Theta^{e}$ la restriction de $\Theta$ à l'ensemble régulier elliptique de $G$.

Définition 1 [9] On dit que $\Theta \in \mathcal{V}(G)$ est un caractère virtuel supertempéré si, pour tout sous-groupe de Cartan $T$ de $G$ et $n$ une constante positive, on a:

$$
\sup _{t \in T^{\prime}}\left|D_{G}(t)\right|^{1 / 2}|\Theta(t)|\left(1+\sigma_{*}(t)\right)^{n}<\infty
$$

où $\sigma_{*}$ estime la croissance sur $G / A_{G}(\operatorname{voir}[13,4.1])$.

Notons $\mathcal{V}_{s t}(G)$, le sous-ensemble de $\mathcal{V}(G)$ formé par les caractères virtuels supertempérés.

\section{Théorème 2 [11]}

1. Le caractère $\Theta \in \mathcal{V}_{s t}(G)$ si et seulement si $\Theta_{P}^{w}=0$ pour tout sous-groupe parabolique propre $P$ de $G$.

2. Soit $\Theta \in \mathcal{V}_{s t}(G)$, si $\Theta^{e}=0$ alors $\Theta=0$.

3. Soient $P_{1}=M N_{1}$ et $P_{2}=M N_{2}$ deux sous-groupes paraboliques de $G$, alors pour tout $\Theta \in \mathcal{V}(G)$, on $a: \Theta_{P_{1}}=\Theta_{P_{2}}$.

On dit que $(M, \sigma)$ est une paire discrète de $G$ si $M \in \mathcal{L}$ et $\sigma \in \prod_{2}(M)$ où $\prod_{2}(M)$ est l'ensemble des classes d'isomorphisme des séries discrètes de $M$. Soit $(M, \sigma)$ une telle paire, notons $W(M):=W^{G}(M)=N_{G}(M) / M$, le groupe de Weyl de $\left(G, A_{M}\right)$, $i_{G, M}(\sigma)$ la classe de la représentation induite $\mathcal{I} n d_{P=M N}(\sigma)$ et $\Re_{\sigma}:=\Re_{\sigma}^{G}$, le $\Re_{\sigma}$-groupe correspondant. Soit $\tilde{\Re}_{\sigma}:=\tilde{\Re}_{\sigma}^{G}$ l'extension centrale du $\Re_{\sigma}$-groupe comme dans $[1, \S 2]$ :

$$
1 \rightarrow Z_{\sigma} \rightarrow \tilde{\Re}_{\sigma} \rightarrow \mathfrak{R}_{\sigma} \rightarrow 1
$$


Il existe un caractère central $\chi_{\sigma}$ de $Z_{\sigma}$ tel que l'ensemble $\Pi_{\sigma}(G)$ des constituants irréductibles de $i_{G, M}(\sigma)$ soit paramétré par l'ensemble $\Pi\left(\tilde{\Re}_{\sigma}, \chi_{\sigma}\right)$ des classes d'équivalence des représentations irréductibles $\rho$ de $\tilde{\Re}_{\sigma}$ ayant $\chi_{\sigma}$ comme $Z_{\sigma^{-}}$caractère central $[1, \S 2]$.

La paramétrisation de $\Pi_{\sigma}(G)$ nous permet de classifier l'ensemble $\Pi(G)$, qui est réunion disjointe des $W_{0}^{G}$-paires discrètes $(M, \sigma)$ des ensembles $\Pi_{\sigma}(G)$ ([8] et $[1, \S 1])$.

Un triplet $(M, \sigma, r)$ est appelée triplet virtuel de $G$ si $(M, \sigma)$ est une paire discrète de $G$ et $r \in \tilde{\Re}_{\sigma}$. A chaque triplet virtuel de $G$, J. Arthur [1, §2] fait correspondre une distribution-caractère $\Theta^{G}(M, \sigma, r):=\Theta(M, \sigma, r)$ appelé caractère virtuel de $G$ qui se décompose sous la forme:

$$
\Theta(M, \sigma, r)=\sum_{\pi \in \Pi_{\sigma}(G)} \theta_{\rho_{\pi}^{\vee}}(r) \Theta_{\pi}
$$

où $\theta_{\rho_{\pi}^{\vee}}$ est le caractère de la contragrédiente de $\rho_{\pi} \in \Pi\left(\tilde{\Re}_{\sigma}, \chi_{\sigma}\right)$ associée à $\pi \in \Pi_{\sigma}(G)$. De plus, on a:

$$
\Theta^{G}\left(w M, w \sigma, w r w^{-1}\right)=\Theta^{G}(M, \sigma, r) \quad \forall w \in W_{0}^{G} .
$$

En inversant (1) on aura, pour tout $\pi \in \Pi_{\sigma}(G)$ :

$$
\Theta_{\pi}=\left|\tilde{\Re}_{\sigma}\right|^{-1} \sum_{r \in \tilde{\Re}_{\sigma}} \theta_{\rho_{\pi}}(r) \Theta^{G}(M, \sigma, r)
$$

ou encore $[1, \S 6]$ :

$$
\Theta_{\pi}=\left|\Re_{\sigma}\right|^{-1} \sum_{r \in \Re_{\sigma}} \theta_{\rho_{\pi}}(r) \Theta^{G}(M, \sigma, r) .
$$

Notons $\mathfrak{a}_{M}$ (resp. $\left.\mathfrak{a}_{G}\right)$, l'algébre de Lie réelle de la composante déployée de $M$ (resp. $G$ ). Le groupe $\mathfrak{R}_{\sigma}$ agit sur $\mathfrak{a}_{M}$. Pour $r \in \Re_{\sigma}$, posons:

$$
\begin{gathered}
\mathfrak{a}_{M}^{r}:=\left\{H \in \mathfrak{a}_{M}: r H=H\right\} \quad \text { et } \quad \mathfrak{a}_{M}^{\Re_{\sigma}}:=\bigcap_{r \in \Re_{\sigma}} \mathfrak{a}_{M}^{r}, \\
\Re_{\sigma, r e g}:=\Re_{\sigma, r e g}^{G}=\left\{r \in \Re_{\sigma} ; \mathfrak{a}_{M}^{r}=\mathfrak{a}_{G}\right\} \\
\mathcal{L}\left(\Re_{\sigma}\right):=\mathcal{L}^{G}\left(\Re_{\sigma}\right)=\left\{S \in \mathcal{L}(M) ; \mathfrak{a}_{S}=\mathfrak{a}_{M}^{r} \text { pour un } r \in \Re_{\sigma}\right\}
\end{gathered}
$$

On dit que le $\Re$-groupe $\Re_{\sigma}$ est essentiel si $\mathfrak{a}_{M}^{\Re_{\sigma}}=\mathfrak{a}_{G}$. Soit $L \in \mathcal{L}(M)$, on dit que $L$ satisfait à la condition de compatibilité d'Arthur si $\mathfrak{a}_{L} \cap \overline{\mathfrak{a}}_{\sigma}^{+}$contient un sous-ensemble ouvert de $\mathfrak{a}_{L}$, où $\mathfrak{a}_{\sigma}^{+}$est la chambre positive correspondante à l'ensemble des racines positives qui annulent la densité de Plancherel [1, §2]. Notons $\mathcal{L}_{A}(M)$ l'ensemble des $L \in \mathcal{L}(M)$ qui satisfait cette condition.

Lemme 3 Soit $(M, \sigma)$ une paire discrète de G. Alors:

(1) Le groupe de réductibilité de $i_{S, M}(\sigma)$ est $\Re_{\sigma}^{S}:=\Re_{\sigma} \cap W^{S}(M)$ pour tout $S \in$ $\mathcal{L}\left(\Re_{\sigma}\right)$.

(2) Le groupe $\Re_{\sigma}$ est une réunion disjointe des ensembles $\Re_{\sigma, \text { reg }}^{S}, S \in \mathcal{L}\left(\Re_{\sigma}\right)$. 
Démonstration D'après J. Arthur [1, §2], si $L \in \mathcal{L}_{A}(M)$, si alors on peut identifier le $\Re$-groupe de $i_{L, M}(\sigma)$ à $\Re_{\sigma} \cap W^{L}(M)$. Par définition, chaque $S \in \mathcal{L}\left(\Re_{\sigma}\right)$ satisfait cette condition de compatibilité; d'où (1). La (2) est claire.

Soit $\tau \in \Pi(L), L \in \mathcal{L}$. Le caractère de la représentation induite $i_{G, L}(\tau)$ sera noté $i_{G, L}\left(\Theta_{\tau}\right)$. Il vérifie l'identité:

$$
i_{G, L}\left(\Theta_{\tau}\right)(f)=\Theta_{\tau}\left(f_{Q}\right), \quad f \in \mathcal{C}(G),
$$

où $f_{Q}$ est le terme constant de $f$ le long d'un parabolique quelconque,

$Q=L N_{Q}$ de $G$. On note souvent:

$$
\Theta_{\tau}\left(f_{L}\right):=\Theta_{\tau}\left(f_{Q}\right)=i_{G, L}\left(\Theta_{\tau}\right)(f), \quad f \in \mathcal{C}(G)
$$

Dans [1, §3], Arthur a montré que les caractères $\Theta^{G}(M, \sigma, r)$ des $W_{0}^{G}$-triplets virtuels $(M, \sigma, r)$ de $G$ forment une base de l'espace $\mathcal{V}(G)$. On se propose, dans la suite, de munir l'espace $\mathcal{V}(G)$ d'une autre base faisant intervenir les caractères virtuels supertempérés.

Théorème 4 Tout élément dans $\mathcal{V}(G)$ s'écrit comme combinaison linéaire finie d'induites de caractères virtuels supertempérés.

Démonstration Soit $\Theta \in \mathcal{V}(G)$. Par définition, il existe $\pi_{1}, \pi_{2}, \ldots, \pi_{k} \in \Pi(G)$ et $c_{i} \in$ $\mathbb{C}$ tel que: $\Theta=\sum_{1 \leq i \leq k} c_{i} \Theta_{\pi_{i}}$. Le théorème sera démontré si on montre que le caractère $\Theta_{\pi}, \pi \in \Pi(G)$ s'écrit comme combinaison linéaire finie d'induites de caractères virtuels supertempérés. En effet, soit $\pi \in \Pi(G)$. On sait que, modulo la conjugaison par $W_{0}^{G}$, il existe une paire discrète unique $(M, \sigma)$ de $G$ telle que $\pi \in \Pi_{\sigma}(G)$. Or, l'égalité (2) nous donne l'expression de son caractère:

$$
\begin{aligned}
\Theta_{\pi} & =\left|\Re_{\sigma}\right|^{-1} \sum_{r \in \Re_{\sigma}} \theta_{\rho_{\pi}}(r) \Theta^{G}(M, \sigma, r) \\
\Theta_{\pi} & =\sum_{S \in \mathcal{L}\left(\Re_{\sigma}\right)}\left|\Re_{\sigma, r e g}^{S}\right|^{-1} \sum_{r \in \Re_{\sigma, r e g}^{S}} \theta_{\rho_{\pi}}(r) \Theta^{G}(M, \sigma, r)
\end{aligned}
$$

d'après le Lemme 3. Par ailleurs, pour $r \in \tilde{\Re}_{\sigma, r e g}^{S}$, le triplet $(M, \sigma, r)$ est un triplet de $S$ et si $r \in \tilde{\Re}_{\sigma, r e g}^{S}$ on a;

$$
\begin{aligned}
\Theta^{G}(M, \sigma, r) & =\sum_{\tau \in \Pi_{\sigma}(S)} \theta_{\rho_{\tau}^{\vee}}(r) i_{G, S}\left(\Theta_{\tau}\right) \\
& =i_{G, S}\left[\sum_{\tau \in \Pi_{\sigma}(S)} \theta_{\rho_{\tau}^{\vee}}(r) \Theta_{\tau}\right] \\
& =i_{G, S}\left[\Theta^{S}(M, \sigma, r)\right] .
\end{aligned}
$$


Par conséquent, l'égalité (3) est équivalente à:

$$
\begin{aligned}
\Theta_{\pi} & =\sum_{S \in \mathcal{L}\left(\Re_{\sigma}\right)}\left|\Re_{\sigma, r e g}^{S}\right|^{-1} \sum_{r \in \Re_{\sigma, \text { reg }}^{S}} \theta_{\rho_{\pi}}(r) i_{G, S}\left(\Theta^{S}(M, \sigma, r)\right) \\
& =\sum_{S \in \mathcal{L}\left(\Re_{\sigma}\right)} i_{G, S}\left[\left|\Re_{\sigma, r e g}^{S}\right|^{-1} \sum_{r \in \Re_{\sigma, r e g}^{S}} \theta_{\rho_{\pi}}(r) \Theta^{S}(M, \sigma, r)\right] \\
& =\sum_{S \in \mathcal{L}\left(\Re_{\sigma}\right)} i_{G, S}\left(\Theta_{\pi, S}\right)
\end{aligned}
$$

où:

$$
\Theta_{\pi, S}:=\left|\Re_{\sigma, r e g}^{S}\right|^{-1} \sum_{r \in \Re_{\sigma, r g}^{S}} \theta_{\rho_{\pi}}(r) \Theta^{S}(M, \sigma, r) .
$$

Or, si $r \in \mathfrak{R}_{\sigma, r e g}^{S}$, le caractère virtuel $\Theta^{S}(M, \sigma, r)$ est dans $\mathcal{V}_{s t}(S)$ [11, Théorème 3.1]. Donc $\Theta_{\pi, S} \in \mathcal{V}_{s t}(S)$. Comme $\Re_{\sigma}$ est fini, les $S \in \mathcal{L}\left(\Re_{\sigma}\right)$ sont en nombre fini. Ceci montre que le caractère d'une représentation irréductible tempérée de $G$ est une combinaison linéaire finie d'induites de caractères virtuels supertempérés. D'où le théorème.

Soit $\Theta \in \mathcal{V}(L), L \in \mathcal{L}$, il existe $\tau_{1}, \tau_{2}, \ldots, \tau_{q} \in \Pi(L)$ et $c_{i} \in \mathbb{C}$ tels que: $\Theta=$ $\sum_{1 \leq i \leq q} c_{i} \Theta_{\tau_{i}}$. Si $t \in W_{0}^{G}$, on note $t \Theta_{\tau_{i}}$ le caractère de la représentation $t \tau_{i} \in \Pi(t L)$ et donc:

$$
t \Theta:=\sum_{1 \leq i \leq q} t \Theta_{\tau_{i}} \quad \in \mathcal{V}(t L)
$$

D'autre part, étant donné que le terme constant $\Theta_{R}$ et le terme constant faible $\Theta_{R}^{w}$ de $\Theta$ le long du sous-groupe parabolique $R=M N_{R}$ de $L$ dépend seulement de $M$ d'après le Théorème 2 (voir aussi [11], p. 154 et (3.3)), on pose: $r_{M, L}(\Theta)=\Theta_{M}:=\Theta_{R}$ et $\Theta_{M}^{w}:=\Theta_{R}^{w}$.

Si $S \in \mathcal{L}$, considérons l'ensemble:

$$
W^{L, S}=\left\{t \in W_{0}^{G} ; t\left(L \cap P_{0}\right) \subset P_{0} \quad \text { et } \quad t^{-1}\left(S \cap P_{0}\right) \subset P_{0}\right\}
$$

D'après [2, 2.12], [11, (3.3)], on a pour tout $\Theta \in \mathcal{V}(L)$ :

$$
\left(i_{G, L}(\Theta)\right)_{S}=\sum_{t \in W^{L, S}} i_{S, S_{t}}\left[(t \Theta)_{S_{t}}\right]
$$

où $S_{t}=S \cap t L$.

\section{Définition 5}

1. On dit que $(L, \Theta)$ est une paire supertempérée de $G$ si $L \in \mathcal{L}$ et $\Theta \in \mathcal{V}_{s t}(L)$.

2. Soient $\left(L_{i}, \Theta_{i}\right)(\mathrm{i}=1,2)$ deux paires supertempérées de $G$. On dit que $\left(L_{1}, \Theta_{1}\right)$ et $\left(L_{2}, \Theta_{2}\right)$ sont conjuguées sous $G$ s'il existe $t \in W_{0}^{G}$ tel que $t L_{1} t^{-1}=L_{2}$ et $t \Theta_{1}=\Theta_{2}$. 
Proposition 6 Soient $\left(L_{1}, \Theta_{1}\right)$ et $\left(L_{2}, \Theta_{2}\right)$ deux paires supertempérées de G. Alors, $i_{G, L_{1}}\left(\Theta_{1}\right)=i_{G, L_{2}}\left(\Theta_{2}\right)$ si et seulement si les paires $\left(L_{1}, \Theta_{1}\right)$ et $\left(L_{2}, \Theta_{2}\right)$ sont conjuguées sous $G$.

Démonstration La démonstration est analogue à celle de Théorème 4 de [7] sur les caractères d'induites cuspidales. En effet pour tout $l_{1} \in\left(L_{1}\right)_{\text {ell }}$, on a:

$$
\left[i_{G, L_{1}}\left(\Theta_{1}\right)\right]_{L_{1}}\left(l_{1}\right)=\left[i_{G, L_{2}}\left(\Theta_{2}\right)\right]_{L_{1}}\left(l_{1}\right)
$$

En utilisant le Théorème 2, l'expression (5) et le fait $t \Theta_{1}=\Theta_{1}$ pour tout $t \in W^{L_{1}, L_{1}}$ tel que $t L_{1}=L_{1}$ [11, Théorème 3.1], l'expression (6) est équivalente à:

$$
\left|t \in W^{L_{1}, L_{1}}: t L_{1}=L_{1}\right| \Theta_{1}\left(l_{1}\right)=\sum_{\left\{t \in W^{\left.L_{2}, L_{1}: t L_{1}=L_{2}\right\}}\right.}\left(t \Theta_{2}\right)\left(l_{1}\right) \quad l_{1} \in\left(L_{1}\right)_{\text {ell }}
$$

Si $L_{1}$ n'est pas conjugué à $L_{2}$, on aura $\Theta_{1}=0$ car elle est nulle sur $\left(L_{1}\right)_{\text {ell }}$ d'après le Théorème 2. Contradiction. D'où $L_{1}$ est conjugué à $L_{2}$. Supposons que $L_{2}=s L_{1}$ pour un certain $s \in W_{0}^{G}$; pour tout $l_{1} \in\left(L_{1}\right)_{\text {ell }}$, l'égalité (6) est équivalente à:

$$
\left|t \in W^{L_{1}, L_{1}}: t L_{1}=L_{1}\right|\left(\Theta_{1}\right)\left(l_{1}\right)=\left|t \in W^{L_{1}, L_{1}}: t L_{1}=L_{1}\right|\left(s^{-1} \Theta_{2}\right)\left(l_{1}\right)
$$

ce qui implique, en utilisant de nouveau le Théorème 2, que: $\Theta_{2}=s \Theta_{1}$.

La réciproque est claire.

Corollaire 7 Soit $\Theta \in \mathcal{V}(G)$, il existe, modulo la conjugaison par $W_{0}^{G}$, une famille finie $\left\{\left(L_{i}, \Theta_{i}\right)\right\}_{1 \leq i \leq k}$ de paires supertempérées de $G$, non conjuguées deux à deux, tel que:

$$
\Theta=\sum_{1 \leq i \leq k} i_{G, L_{i}}\left(\Theta_{i}\right)
$$

Démonstration Se déduit de la Proposition 6 et du Théorème 4.

Soient $\pi \in \Pi(G)$ et $(L, \Theta)$ une paire supertempérée de $G$. On écrit $\Theta_{\pi} \hookrightarrow i_{G, L}(\Theta)$ si $\Theta_{\pi}$ apparaît dans la décomposition de $i_{G, L}(\Theta)$.

Théorème 8 Soient $\left(L_{i}, \Theta_{i}\right) \quad i=1,2$ deux paires supertempérées de $G$ et $\pi \in \Pi(G)$. Si $\Theta_{\pi}$ apparaît dans la décomposition de $i_{G, L_{1}}\left(\Theta_{1}\right)$ et $i_{G, L_{2}}\left(\Theta_{2}\right)$ alors il existe $t \in W_{0}^{G}$ tel que $t L_{1}=L_{2}$. De plus $t \Theta_{1}$ apparaît dans la décomposition de $\Theta_{2}$.

Démonstration Si $\Theta_{\pi} \hookrightarrow i_{G, L_{2}}\left(\Theta_{2}\right)$ alors, il est clair que $w \Theta_{2} \hookrightarrow r_{L_{2}, G}\left(\Theta_{\pi}\right)$, pour un certain $w \in W_{0}^{L_{2}}$. Ainsi:

$$
w \Theta_{2} \hookrightarrow r_{L_{2}, G}\left(\Theta_{\pi}\right) \hookrightarrow r_{L_{2}, G} \circ i_{G, L_{1}}\left(\Theta_{1}\right)
$$

En appliquant l'expression (5), on retrouve qu'il existe $t \in W_{0}^{G}$ vérifiant $t L_{1} \subset L_{2}$ tel que:

$w \Theta_{2} \hookrightarrow i_{L_{2}, t L_{1}}\left(t \Theta_{1}\right)$, or ceci implique que $t \Theta_{1} \hookrightarrow r_{t L_{1}, L_{2}}\left(w \Theta_{2}\right)=r_{t L_{1}, L_{2}}\left(\Theta_{2}\right)$. Comme $\Theta_{2} \in \mathcal{V}_{s t}\left(L_{2}\right)$, on déduit qu'ils existent $t \in W_{0}^{G}$ tel que $t L_{1}=L_{2}$ et $t \Theta_{1} \hookrightarrow \Theta_{2}$. Mais ceci implique $t \Theta_{1}$ apparaît dans la décomposition de $\Theta_{2}$. 
Corollaire 9 [9] Soient $\left(M_{1}, \sigma_{1}\right)$ et $\left(M_{2}, \sigma_{2}\right)$ deux paires discrètes de $G$. Si $\Pi_{\sigma_{1}}(G)$ et $\Pi_{\sigma_{2}}(G)$ possèdent un constituant en commun alors il existe $t \in W_{0}^{G}$ tel que $t M_{1}=M_{2}$ et $t \sigma_{1}=\sigma_{2}$. Réciproquement s'il existe $t \in W_{0}^{G}$ tel que $t M_{1}=M_{2}$ et $t \sigma_{1}=\sigma_{2}$ alors $\Pi_{\sigma_{1}}(G)=\Pi_{\sigma_{2}}(G)$.

Démonstration Comme $\Theta_{\sigma} \in \mathcal{V}_{s t}(G)$ si et seulement si $\sigma \in \Pi_{2}(G)$, [11, Corollaire 2.6], le résultat se déduit du théorème 8 . La réciproque est claire.

Terminons ce paragraphe par un résultat sur les traces compactes.

Notons par $P_{t}=M_{t} N_{t}$ le sous-groupe parabolique de $G$ correspondant à l'élément semi-simple $t$ de $G$ [6] et $G_{c}$ l'ensemble des éléments semi-simples $t$ de $G$ tel que $P_{t}=G$. L'ensemble $G_{c}$ est appelé "la partie compacte" de $G$.

Lemme 10 Soit $\Theta \in \mathcal{V}_{s t}(G)$. Si $t \notin G_{c} \cap G^{\prime}$ alors $\Theta(t)=0$.

Démonstration Pour tout $t \in G^{\prime}$, on a [3]: $\Theta(t)=\Theta_{P_{t}}(t)$ où $\Theta_{P_{t}}$ est le terme constant de $\Theta$ le long de $P_{t}$. Si $P_{t}$ est un sous-groupe parabolique propre de $G$, on aura $\Theta(t)=0$ car $\Theta \in \mathcal{V}_{s t}(G)$. Autrement dit, $\Theta(t)$ est nul sauf si $P_{t}=G$. D'où le lemme.

Soient $\Theta \in \mathcal{V}(G)$ et $f \in \mathcal{C}(G)$. La trace compacte de $\Theta$ est définie par:

$$
<\Theta, f>_{G, c}:=\int_{G_{c}} \Theta(x) f(x) d x .
$$

Remarquons que le Lemme 10 implique que si $\Theta \in \mathcal{V}_{s t}(G)$ alors:

$$
<\Theta, f>=<\Theta, f>_{G, c}, \quad f \in \mathcal{C}(G) \text {. }
$$

Corollaire 11 Si $\Theta \in \mathcal{V}(G)$ alors il existe une famille finie de paires supertempérées $\left\{\left(L_{i}, \Theta_{i}\right)\right\}_{1 \leq i \leq p}$, de $G$, telle que

$$
<\Theta, f>=\sum_{1 \leq i \leq p}<\Theta_{i}, f_{L_{i}}>_{L_{i}, c} \quad f \in \mathcal{C}(G)
$$

Démonstration Soit $\Theta \in \mathcal{V}(G)$. Le Corollaire 7 implique que, modulo conjugaison par $W_{0}^{G}$, il existe une famille finie $\left\{\left(L_{i}, \Theta_{i}\right)\right\}_{1 \leq i \leq p}$ telle que:

$$
<\Theta, f>=\sum_{1 \leq i \leq p} i_{G, L_{i}}\left(\Theta_{i}\right)(f), \quad f \in \mathcal{C}(G) .
$$

Or, pour tout $1 \leq i \leq p$, et tout $Q_{i}$ sous-groupe parabolique de $G$ ayant $L_{i}$ comme composante de Lévi, on a:

$$
<\Theta_{i}, f_{L_{i}}>_{L_{i}, c}=\Theta_{i}\left(f_{L_{i}}\right)=\Theta_{i}\left(f_{Q_{i}}\right)=i_{G, L_{i}}\left(\Theta_{i}\right)(f)
$$

où $f_{Q_{i}}$ est le terme constant de $f$ le long de $Q_{i}$, d'où le corollaire. 
Le Corollaire 11 est un résultat plus fin, dans le cas tempéré, que la Proposition 1 de Clozel [4].

\section{Classification de $\Pi(G)$}

La classification de $\Pi_{\sigma}(G)$ à travers $\Pi\left(\tilde{\Re}_{\sigma}, \chi_{\sigma}\right)$ fait intervenir beaucoup de choix, pour cela on se propose de classifier, dans ce paragraphe, $\Pi(G)$ à travers les limites de séries discrètes. Cette classification a été énoncée par Clozel comme conjecture [5].

Définition 12 Une représentation dans $\Pi(G)$ est dite essentielle (ou limite de série discrète [5]) si elle n'est pas proprement irréductiblement induite par induction parabolique. Notons:

$$
\Pi_{\text {ess }}(G):=\{\pi \in \Pi(G) \quad / \quad \pi \quad \text { est essentielle }\}
$$

A l'aide de ce qui précède, $\Pi_{e s s}(G)$ est une réunion disjointe sur les $W_{0}^{G}$-paires discrètes $(M, \sigma)$ des ensembles:

$$
\Pi_{\sigma, e s s}(G):=\Pi_{\sigma}(G) \cap \Pi_{e s s}(G) .
$$

Le $\Re_{\sigma}$-groupe de $i_{G, M}(\sigma)$ est dit essentiel si $\mathfrak{a}_{M}^{\Re_{\sigma}}=\mathfrak{a}_{G}$.

Théorème 13 Soient $(M, \sigma)$ une paire discrète de $G$ et $\pi \in \Pi_{\sigma}(G) . \pi \in \Pi_{\sigma, \text { ess }}(G)$ si et seulement si le groupe $\Re_{\sigma}$ est essentiel.

Démonstration Si le groupe $\Re_{\sigma}$ n'est pas essentiel, alors il existe $L \in \mathcal{L}_{0}(M)$ tel que $\mathfrak{a}_{M}^{\Re_{\sigma}}=\mathfrak{a}_{L}$ et donc tout élément de $\Re_{\sigma}$ laisse invariant point par point $\mathfrak{a}_{L}$, or ceci signifie que $\Re_{\sigma}=\Re_{\sigma}^{L}$, car $\Re_{\sigma}^{L}=\Re_{\sigma} \cap W^{L}(M)$. Mais $\left|\Pi\left(\tilde{\Re}_{\sigma}, \chi_{\sigma}\right)\right|=\left|\Pi_{\sigma}(G)\right|$ et $\left|\Pi\left(\tilde{\Re}_{\sigma}^{L}, \chi_{\sigma}\right)\right|=\left|\Pi_{\sigma}(L)\right|$. Ainsi $\Re_{\sigma}=\Re_{\sigma}^{L}$ implique $\left|\Pi_{\sigma}(G)\right|=\left|\Pi_{\sigma}(L)\right|$ et donc chaque composante irréductible de $i_{G, M}(\sigma)$ est de la forme $i_{G, L}(\tau)$ pour $\tau \in \Pi_{\sigma}(L)$.

Réciproquement. Supposons que $\Pi_{\sigma}(G)$ a un élément proprement irréductiblement induit, i.e., il existe $\pi \in \Pi_{\sigma}(G)$ tel que $\pi=i_{G, L}(\tau)$ où $\tau \in \Pi(L)$ et $L \in \mathcal{L}_{0}$.

$\tau \in \Pi(L)$ implique qu'il existe, modulo conjugaison par $W_{0}^{L}$, une unique paire discrète $\left(M_{1}, \sigma_{1}\right)$ de $L$ tel que $\tau \in \Pi_{\sigma_{1}}(L)$. Dans ce cas, $\pi \in \Pi_{\sigma}(G) \cap \Pi_{\sigma_{1}}(G)$ et alors il existe $t \in W_{0}^{G}$ tel que $(M, \sigma)=\left(t M_{1}, t \sigma_{1}\right)$ (Corollaire 9). Si on suppose que $(M, \sigma)=\left(M_{1}, \sigma_{1}\right)$, alors $L \in \mathcal{L}_{0}(M)$ et $\tau \in \Pi_{\sigma}(L)$. Sans perte de généralité, supposons que $L$ satisfait la condition de compatibilité d'Arthur, i.e., $L \in \mathcal{L}_{0}(M) \cap$ $\mathcal{L}_{A}(M)$.

Le Théorème 4 et l'expression (4) impliquent que le caractère de $\pi=$ $\pi_{\rho}, \quad \rho \in \Pi\left(\tilde{\Re}_{\sigma}, \chi_{\sigma}\right)$ s'écrit comme combinaison linéaire finie d'induites de supertempérées, i.e.,

$$
\Theta_{\pi}=\sum_{S \in \mathcal{L}\left(\Re_{\sigma}\right)} i_{G, S}\left(\Theta_{\pi, S}\right)
$$

où, pour $S \in \mathcal{L}\left(\Re_{\sigma}\right)$, la paire $\left(S, \Theta_{\pi, S}\right)$ est une paire supertempérée de $G$. 
De même, le caractère de $\tau=\tau_{\rho_{L}}, \quad \rho_{L} \in \Pi\left(\tilde{\Re}_{\sigma}^{L}, \chi_{\sigma}\right)$ s'écrit comme combinaison linéaire finie d'induites de supertempérées, i.e.,

$$
\Theta_{\tau}=\sum_{T \in \mathcal{L}^{L}\left(\Re_{\sigma}^{L}\right)} i_{L, T}\left(\Theta_{\tau, T}\right)
$$

où, pour $T \in \mathcal{L}^{L}\left(\Re_{\sigma}^{L}\right)$, la paire $\left(T, \Theta_{\tau, T}\right)$ est une paire supertempérée de $L$.

Ce qui implique, par transitivité de l'induction, que:

$$
\Theta_{\pi}=i_{G, L}\left(\Theta_{\tau}\right)=\sum_{T \in \mathcal{L}^{L}\left(\Re_{\sigma}^{L}\right)} i_{G, T}\left(\Theta_{\tau, T}\right) .
$$

Mais le Corollaire 7, implique que la famille $\left\{\left(S, \Theta_{\pi, S}\right)\right\}_{S \in \mathcal{L}\left(\Re_{\sigma}\right)}$ est conjuguée à la famille $\left\{\left(T, \Theta_{\tau, T}\right)\right\}_{T \in \mathcal{L}^{L}\left(\Re_{\sigma}^{L}\right)}$.

En particulier, la famille $\left\{S ; S \in \mathcal{L}\left(\Re_{\sigma}\right)\right\}$ est conjuguée à la famille $\{T ; \quad T \in$ $\left.\mathcal{L}^{L}\left(\Re_{\sigma}^{L}\right)\right\}$. Ainsi, si on suppose que ces deux familles sont égales, on obtient:

$\mathfrak{a}_{G} \neq \mathfrak{a}_{L} \subset \mathfrak{a}_{S} \quad$ pour tout $S \in \mathcal{L}\left(\Re_{\sigma}\right)$ et alors:

$$
\mathfrak{a}_{G} \neq \mathfrak{a}_{L} \subset \bigcap_{S \in \mathcal{L}\left(\mathfrak{\Re}_{\sigma}\right)} \mathfrak{a}_{S}=\bigcap_{r \in \mathfrak{H}_{\sigma}} \mathfrak{a}_{M}^{r}=\mathfrak{a}_{M}^{\mathfrak{\Re}_{\sigma}}
$$

D’où le théorème.

Le Théorème 13 est une généralisation du Lemme 1.3 de R. Herb [10].

Corollaire 14 (Conjecture [5]) Soit $(M, \sigma)$ une paire discrète de G. Supposons que:

$$
i_{G, M}(\sigma)=\pi_{1} \oplus \pi_{2} \oplus \ldots \ldots \ldots \oplus \pi_{n}
$$

Si $\pi_{1} \in \Pi_{\text {ess }}(G)$ alors tous les $\pi_{i} \in \Pi_{\text {ess }}(G)$.

Démonstration Se déduit du Théorème 13.

Proposition 15 (Conjecture [5]) Toute représentation irréductible tempérée de G est irréductiblement induite d'une essentielle.

Démonstration Soit $\pi \in \Pi(G)$, on sait qu'il existe, modulo conjugaison par $W_{0}^{G}$, une unique paire discrète $(M, \sigma)$ de $G$ telle que $\pi \in \Pi_{\sigma}(G)$. On va distinguer deux cas:

Si $\mathfrak{a}_{M}^{\Re_{\sigma}}=\mathfrak{a}_{G}$, alors, le Théorème 13 implique que, $\pi \in \Pi_{\sigma, \text { ess }}(G)$.

Si $\mathfrak{a}_{M}^{\Re_{\sigma}} \neq \mathfrak{a}_{G}$, alors, aussi le Théorème 13 implique qu'il existe $L \in \mathcal{L}_{0}(M)$ et $\tau \in$ $\Pi_{\sigma}(L)$ tel que $\pi$ est proprement irréductiblement de $\tau$.

Mais $\mathfrak{a}_{M}^{\Re_{\sigma}}=\mathfrak{a}_{L}$ implique que $\mathfrak{R}_{\sigma}=\mathfrak{R}_{\sigma}^{L}$ et alors $\mathfrak{a}_{M}^{\Re_{\sigma}^{L}}=\mathfrak{a}_{L}$, ce qui signifie que $\mathfrak{R}_{\sigma}^{L}$ est essentiel et donc le Théorème 13 appliqué à $L$ implique $\tau \in \Pi_{\sigma, e s s}(L)$. D'où la proposition. 
Corollaire 16 Soit $\pi \in \Pi(G)$, si:

$$
\begin{array}{lll}
\pi=i_{G, L_{1}}\left(\delta_{1}\right) & \delta_{1} \in \Pi_{\text {ess }}\left(L_{1}\right) & L_{1} \in \mathcal{L} \\
\pi=i_{G, L_{2}}\left(\delta_{2}\right) & \delta_{2} \in \Pi_{\text {ess }}\left(L_{2}\right) & L_{2} \in \mathcal{L}
\end{array}
$$

alors il existe $t \in G$ tel que $t L_{1}=L_{2}$ et $t \delta_{1}=\delta_{2}$.

Démonstration Si $\delta_{1} \in \Pi_{\text {ess }}\left(L_{1}\right)$ alors le Théorème 13, implique qu'il existe une paire discrète $\left(M_{1}, \sigma_{1}\right)$ de $L_{1}$ vérifiant $\mathfrak{a}_{M_{1}}^{\Re_{\sigma_{1}}^{L_{1}}}=\mathfrak{a}_{L_{1}}$ tel que $\delta_{1} \in \Pi_{\sigma_{1}}\left(L_{1}\right)$. Mais comme $\pi$ est irréductiblement induite de $\delta_{1}$, on aura $\Re_{\sigma_{1}}=\mathfrak{R}_{\sigma_{1}}^{L_{1}}$, ce qui revient à ce que $\mathfrak{a}_{M_{1}}^{\Re_{\sigma_{1}}}=\mathfrak{a}_{L_{1}}$. De même $\delta_{2} \in \Pi_{\text {ess }}\left(L_{2}\right)$, implique qu'il existe une paire discrète $\left(M_{2}, \sigma_{2}\right)$ de $L_{2}$ vérifiant $\mathfrak{a}_{M_{2}}^{\Re_{\sigma_{2}}}=\mathfrak{a}_{L_{2}}$ tel que $\delta_{2} \in \Pi_{\sigma_{2}}\left(L_{2}\right)$. Ainsi $\pi=i_{G, L_{1}}\left(\delta_{1}\right)=i_{G, L_{2}}\left(\delta_{2}\right)$ implique $\pi \in \Pi_{\sigma_{1}}(G) \cap \Pi_{\sigma_{2}}(G)$ et donc il existe $t \in G$ tel que $\left(M_{1}, \sigma_{1}\right)=\left(t M_{2}, t \sigma_{2}\right)$ d'après le corollaire 9. Supposons que $\left(M_{1}, \sigma_{1}\right):=\left(M_{1}, \sigma_{1}\right)=\left(M_{2}, \sigma_{2}\right)$, alors on aura $\mathfrak{a}_{L}:=$ $\mathfrak{a}_{L_{1}}=\mathfrak{a}_{L_{2}}$ et donc $\delta_{1}=\delta_{2} \operatorname{car}\left|\Pi_{\sigma}(G)\right|=\left|\Pi_{\sigma}(L)\right|$.

Open Access This article is distributed under the terms of the Creative Commons Attribution Noncommercial License which permits any noncommercial use, distribution, and reproduction in any medium, provided the original author(s) and source are credited.

\section{References}

1. Arthur, J.: On elliptic tempered characters. Acta Math. 171, 73-138 (1993)

2. Bernstein, J., Zelevinsky, Z.; Induced representations of reductive p-adic groups I. Ann. Sci. Ec. Norm. Super. 4ème Série. 10, 441-472 (1977)

3. Casselman, W.: Characters and Jacquet modules. Math. Ann. 230,101-105 (1977)

4. Clozel, L.: Invariant analysis on the Schwartz space of a reductive p-adic group. In: Harmonic Analysis on Reductive Groups, Progress in Mathematics, vol. 101, pp. 101-121. Birkhauser, Boston (1991)

5. Clozel, L.: Orbital integrals on p-adics groups. A proof of the Howe conjecture. Ann. Math. 129, 237-251 (1989)

6. Deligne, P.: Le support du caractère d'une représentation supercuspidale. C. R. Acad. Sci. Paris Ser A-B 283(4), Aii, A155-A157 (1976)

7. Van Dijk, G.: Computation of certain induced characters of $p$-adic groups. Math. Ann. 199, 229$240(1972)$

8. Harish-Chandra: The Plancherel formula for reductive p-adic groups. In: Collected Papers, vol IV, pp. 353-367. Springer, New York (1984)

9. Harish-Chandra: Supertempered distributions on real reductive groups. Studies in applied math. Adv. Math. Supp. Stud. 8, 139-158 (1983)

10. Herb, R.: Elliptic representations for $S p(2 n)$ et $S O(n)$. Pac. J. Math. 161, 347-358 (1993)

11. Herb, R.: Supertempered virtual characters. Compos. Math. 93, 139-154 (1994)

12. Kazhdan, D.: Cuspidal geometry of p-adic groups. J. Anal. Math. 47, 1-36 (1986)

13. Silberger, A.J.: Introduction to harmonic analysis on reductive p-adic groups. In: Mathematical Notes, no. 23. Princeton University Press, Princeton (1979) 